\title{
Long non-coding RNA CCDC144NL-AS1 sponges miR-143-3p and regulates MAP3K7 by acting as a competing endogenous RNA in gastric cancer
}

\author{
Hao Fan', Yugang Ge $\mathbb{D}^{1}$, Xiang Ma', Zengliang Li', Liang Shi (1)', Linling Lin $\mathbb{D}^{1}$, Jian Xiao', Wangwang Chen', \\ Peidong $\mathrm{Ni}^{1}$, Li Yang $\mathbb{1}^{1,2}$ and Zekuan $\mathrm{Xu}^{1}$
}

\begin{abstract}
Gastric cancer (GC) has been one of the most leading cause of cancer-death worldwide. Long non-coding RNAs (IncRNAs) have been found to be related with the carcinogenesis and the development of various cancers, including GC. However, there are still many GC-related IncRNAs functional roles and molecular mechanisms that have not yet been clearly studied. Herein, we report InCRNA CCDC144NL-AS1, which has not been explored in GC, and it is markedly upregulated in GC tissues, which may serve as an independent predictor of poor prognosis. We found that CCDC144NL-AS1 expression was significantly positively associated with a larger tumor size and more pronounced lymph node metastasis. Through a series of in vivo and in vitro functional experiments, we observed that CCDC144NLAS1 could facilitate cell proliferation, invasion and migration and inhibit cell apoptosis in GC. Further mechanism investigation revealed that CCDC144NL-AS1 acted as a competing endogenous RNA (ceRNA) for sponging miR-143-3p and upregulated the expression of its direct endogenous target MAP3K7 in GC. Taken together, our results elucidate the oncogenic roles of CCDC144NL-AS1/miR-143-3p/MAP3K7 axis in GC progression, providing inspiration for further understanding of the mechanism of GC and making CCDC144NL-AS1 as a potential novel diagnostic and therapeutic target for GC.
\end{abstract}

\section{Introduction}

Gastric cancer (GC) ranks among the highest malignancies, and it is also an important risk factor that endangers human health worldwide. Its morbidity and mortality are high, and the lack of effective treatments leads to a very poor prognosis ${ }^{1,2}$. Although in recent decades we have made some progress in fields such as surgical techniques, immunotherapy and molecular targeted therapy, the therapeutic effect is still not very satisfactory, which is attributed to the fact that we know

\footnotetext{
Correspondence: Li Yang (pwkyangli@njmu.edu.cn)

'Department of General Surgery, the First Affiliated Hospital of Nanjing Medical University, Nanjing, Jiangsu Province, China

${ }^{2}$ Department of General Surgery, Liyang People's Hospital, Liyang Branch Hospital of Jiangsu Province Hospital, Liyang, Jiangsu Province, China

These authors contributed equally: Hao Fan, Yugang Ge, Xiang Ma, Zengliang Li

Edited by G. Ciliberto
}

little about the specific mechanism underlying the development of gastric carcinogenesis at the cellular and molecular level ${ }^{3-5}$. Therefore, it is significant meaningful to elucidate the molecular mechanism of the pathogenesis of GC and to find promising and effective diagnostic and therapeutic target for GC.

The Encyclopedia of DNA Elements (ENCODE) Project Consortium has revealed that the majority of the human genome are transcribed into non-coding RNAs (ncRNAs), while protein-coding genes account for only $2 \%^{6}$. Long non-coding RNAs (lncRNAs) share limited or no proteincoding capacity and have transcripts of more than 200 nucleotides in length ${ }^{7}$. LncRNAs usually play their biological functions by directly or indirectly regulating the expression of potential target genes at epigenetic modification, transcriptional and post-transcriptional levels ${ }^{8,9}$. These biological processes include cell proliferation,

\section{(c) The Author(s) 2020}

(c) (i) Open Access This article is licensed under a Creative Commons Attribution 4.0 International License, which permits use, sharing, adaptation, distribution and reproduction cc in any medium or format, as long as you give appropriate credit to the original author(s) and the source, provide a link to the Creative Commons license, and indicate if changes were made. The images or other third party material in this article are included in the article's Creative Commons license, unless indicated otherwise in a credit line to the material. If material is not included in the article's Creative Commons license and your intended use is not permitted by statutory regulation or exceeds the permitted use, you will need to obtain permission directly from the copyright holder. To view a copy of this license, visit http://creativecommons.org/licenses/by/4.0/. 


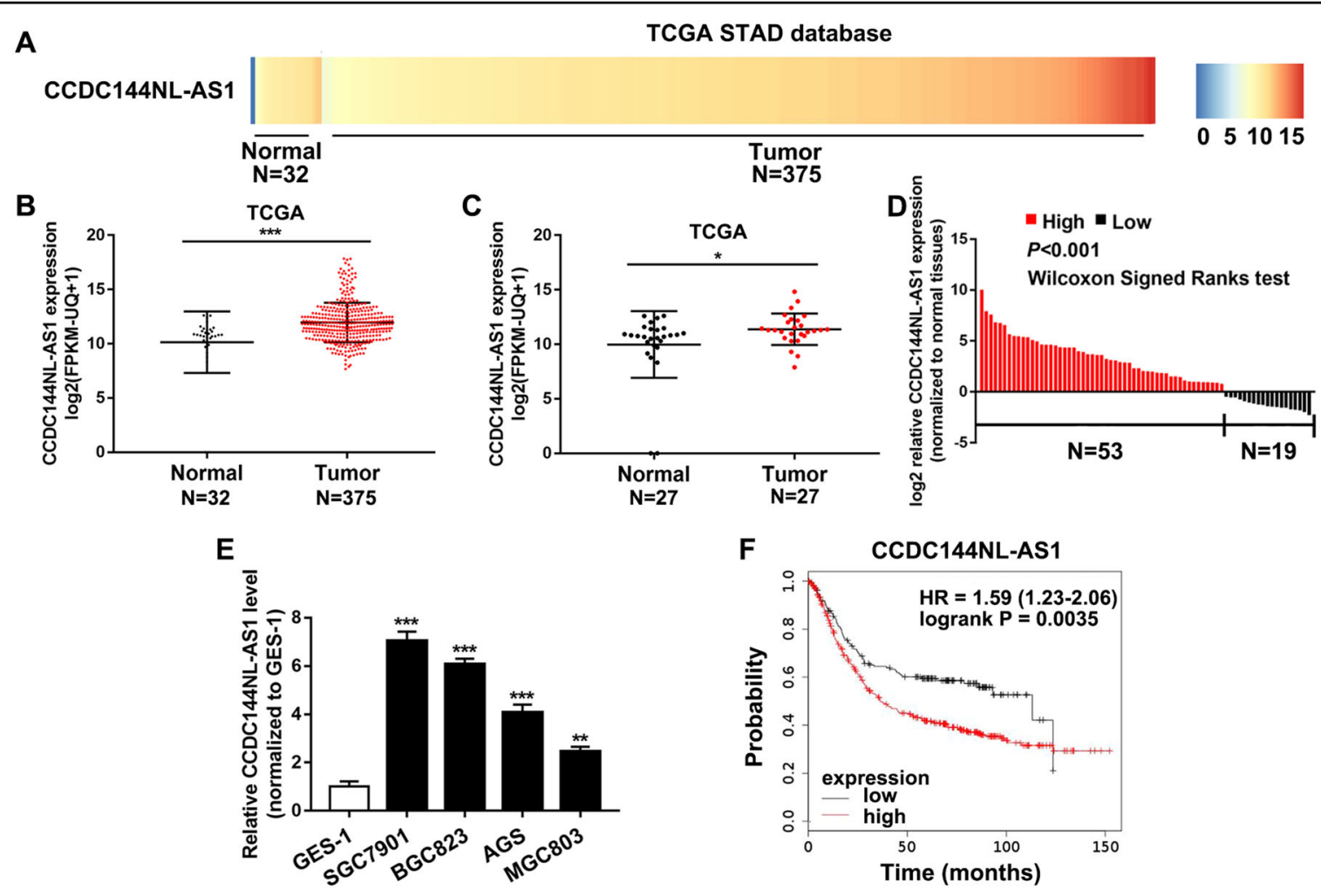

Fig. 1 CCDC144NL-AS1 is upregulated in GC and associated with poor prognosis. a Heat map analysis of CCDC144NL-AS1 expression in GC from the TCGA database. b, c Relative expression of CCDC144NL-AS1 in unpaired and paired GC tissues was analyzed from the TCGA database. d qRT-PCR was used to detect the expression of CCDC144NL-AS1 in 72 GC tissues and paired adjacent normal tissues. e qRT-PCR analysis of CCDC144NL-AS1 expression in GC cells and normal gastric epithelium cell line (GES-1). f Online Kaplan-Meier overall survival (OS) curves according to CCDC144NL-AS1 expression levels. ${ }^{*} p<0.05,{ }^{* *} p<0.01,{ }^{* *} p<0.001$.

differentiation, and metastasis ${ }^{10}$. A growing number of studies show that dysregulation of lncRNAs are related with the development and the carcinogenesis of many tumors, including $\mathrm{GC}^{11-14}$. Recently, lncRNAs have been widely reported to function as competing endogenous RNA (ceRNA), ceRNA can affect gene silencing caused by microRNA through binding microRNA response elements (MREs), which reveals the existence of a RNAmicroRNA regulatory pathway and has great biological significance ${ }^{15-17}$. Construction and analysis of IncRNAmediated ceRNA network has opened a new way for understanding the pathogenesis of GC and finding novel diagnostic biomarkers or potential therapeutic targets for GC.

In the present study, we found a GC-associated lncRNA CCDC144NL-AS1, which was markedly upregulated in GC tissues and related with poor prognosis, while its function in GC has not been reported. Through a series of in vitro and in vivo functional experiments, we found that CCDC144NL-AS1 could facilitate cell proliferation, invasion and migration in GC. With the deepening of research, we discovered that CCDC144NL-AS1 might regulate MAP3K7 expression by sponging miR-143-3p to exert ceRNA function. Taken together, our study elucidates oncogenic roles of CCDC144NL-AS1/miR-143-3p/ MAP3K7 axis in GC tumorigenesis, which may provide a prognostic marker as well as a promising therapeutic target for GC patients.

\section{Results}

CCDC144NL-AS1 is upregulated in GC and associated with poor prognosis

By analyzing TCGA STAD database including RNA sequencing (RNA-seq) data of $375 \mathrm{GC}$ tissues and 32 adjacent non-tumor tissues, CCDC144NL-AS1 was identified as GC-associated IncRNA that may be related with gastric tumorigenesis. As a result, we took heat map analysis and found that the gene expression of CCDC144NL-AS1 was markedly elevated in GC patients, which suggested that CCDC144NL-AS1 might play huge roles in GC (Fig. 1a). We retrieved TCGA STAD database and discovered that CCDC144NL-AS1 mRNA expression was markedly overexpressed both in unpaired and paired GC tissues (Fig. 1b, c). Then, we detected the mRNA expression of CCDC144NL-AS1 in our own 72 paired GC tissues and cell lines, which suggested a high expression of 
CCDC144NL-AS1 in GC (Fig. 1d, e). SGC7901 and BGC823 cell lines with the highest CCDC144NL-AS1 expression levels were selected for subsequent experiments. The clinicopathologic features of the GC patients showed that high CCDC144NL-AS1 expression was obviously related with a larger tumor size and more profound lymphatic metastasis rate (Table S1).Further, the online Kaplan-Meier plot (http://www.kmplot.com) indicated that a high CCDC144NL-AS1 expression level was correlated with poor overall survival (Fig. 1f). These results revealed that CCDC144NL-AS1 was upregulated in GC and related with poor prognosis.

\section{Altering the expression of CCDC144NL-AS1 affects the proliferation, migration and invasion of GC cells}

To explore the biological functions of CCDC144NLAS1 in GC cells, we knocked down CCDC144NL-AS1 expression by transfecting with siRNAs in BGC823 and SGC7901 cells (Fig. 2a). CCK-8 proliferation experiments suggested that silencing CCDC144NL-AS1 could dramatically attenuate cell proliferation ability compared with the negative control (NC) (Fig. 2b, c). Colony formation assay indicated that downregulation of CCDC144NL-AS1 significantly reduced the cell colony ability of SGC7901 and BGC823 cells (Fig. 2d). Likewise, Edu assays showed the same results that CCDC144NL-AS1 silencing inhibits GC cell proliferation (Fig. 2e-g). Furthermore, transwell assays demonstrated that CCDC144NL-AS1 knockdown repressed the migration and invasion of GC cells (Fig. $2 \mathrm{~h}-\mathrm{k}$ ). Moreover, in the wound healing assays, silencing CCDC144NL-AS1 attenuated the migration rate of GC cells (Fig. 2l-n).

In addition, we upregulated CCDC144NL-AS1 by transfecting pcDNA-CCDC144NL-AS1 into SGC7901 and BGC823 cells and the expression level of CCDC144NL-AS1 increased (Fig. S1A). CCK-8 assays, colony formation assays and Edu assays revealed that overexpression of CCDC144NL-AS1 could promote SGC7901 and BGC823 cells proliferation (Fig. S1B-G). Furthermore, transwell assays suggested that the cell numbers of migrated and invaded cells increased by overexpressing CCDC144NL-AS1 (Fig. S1H-K). Wound healing assays showed the same results as transwell migration assays (Fig. S1L-N). These results suggested that silencing CCDC144NL-AS1 inhibited the proliferation, migration and invasion of GC cells and overexpression of CCDC144NL-AS1 had the opposite effects. CCDC144NL-AS1 may play a key role as an oncogene in GC.

\section{Knockdown of CCDC144NL-AS1 induces cell apoptosis, inhibits GC tumor growth and metastasis in vivo}

The cell apoptosis is an important factor which could influence GC cell proliferation. Flow cytometric assays revealed that knockdown of CCDC144NL-AS1 induced SGC7901 and BGC823 cells apoptosis (Fig. 3a-c). Subsequently, we inoculated subcutaneously the SGC7901 cells stably transfected with shRNA targeting CCDC144NL-AS1 or their negative control groups into female nude mice to determine whether CCDC144NLAS1 affected tumor growth in vivo, we discovered that the average tumor size and weight generated by CCDC144NL-AS1 knockdown group were markedly smaller than those generated from their control group (Fig. 3d-f). Tumor tissues harvested from nude mice was used to detect the expression level of proliferation marker Ki-67 by IHC staining, it suggested that the Ki-67 expression was lower in sh-CCDC144NL-AS1 group than control group (Fig. 3g). To explore the function of CCDC144NL-AS1 on tumor metastasis in vivo, stable transfected cells treated differently were injected into tail vein of $B A L B / c$ nude mice separately. The results suggested that lung metastasis in CCDC144NL-AS1 knockdown group was alleviated (Fig. $3 \mathrm{~h}$ ). These results showed that silencing CCDC144NL-AS1 induced cell apoptosis, inhibited GC tumor growth and metastasis in vivo.

\section{CCDC144NL-AS1 acts as a molecular sponge for miR-143- $3 p$ in GC cells}

To explore how CCDC144NL-AS1 exerts its function, we predicted its subcellular localization by IncATLAS (http://lncatlas.crg.eu/). The result showed that CCDC144NL-AS1 was mainly located in the cytoplasm of all the available cell types (Fig. 4a). FISH assay and quantitative RT-PCR analysis suggested that CCDC144NL-AS1 was mainly located in the cytoplasm of GC cells (Fig. 4b, c). These results indicated that CCDC144NL-AS1 may function as a ceRNA of miRNAs. Then, we predicted the potential miRNAs that may interact with CCDC144NL-AS1 using DIANA (http:// carolina.imis.athena-innovation.gr/diana_tools/web/ index.php? $\mathrm{r}=$ site\%2Ftools) and lncRNASNP2 (http:// bioinfo.life.hust.edu.cn/lncRNASNP/\#!/) database and observed that CCDC144NL-AS1 sequence contains potential binding sites of several miRNAs such as miR143-3p, miR-130a-5p, miR-874-3p, miR-551b-5p, miR383-3p (Fig. 4d). We then explored the effect of downregulation or upregulation of CCDC144NL-AS1 on the expression level of these miRNAs and found that miR143-3p was the most changed one (Fig. 4e, f). A dualluciferase reporter assay showed that miR-143-3p significantly reduced the relative luciferase activity of the wild-type CCDC144NL-AS1 3'UTR (Fig. 4g, h). TCGA database has revealed that miR-143-3p was downregulated in GC tissues, which suggested that miR-143-3p might function as a tumor suppressor gene (Fig. 4i). Consistent with TCGA analysis results, we verified lower miR-143-3p expression in 72 GC tissues (Fig. 4j). A 


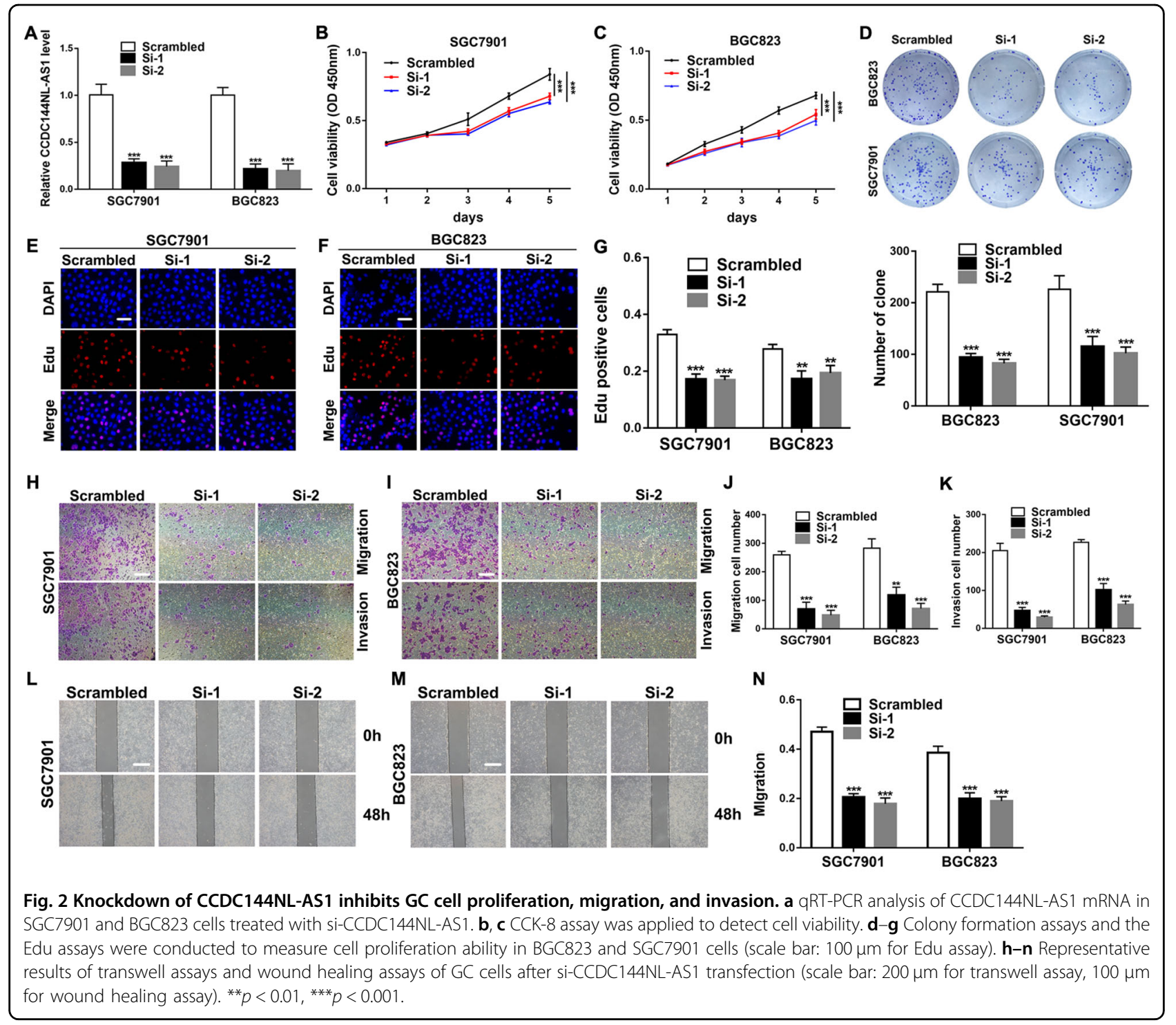

negative correlation was also found between CCDC144NL-AS1 and miR-143-3p expression in 72 pairs of GC samples (Fig. 4k). These results indicated that CCDC144NL-AS1 directly "sponges" miR-143-3p.

The regulation of CCDC144NL-AS1 on GC cells is mediated by miR-143-3p

To further explore the biological interactions between CCDC144NL-AS1 and miR-143-3p in GC, SGC7901, and BGC823 cells were co-transfected with si-CCDC144NLAS1 and miR-143-3p inhibitor. From CCK-8 assays, colony formation assays, Edu assays, and wound healing assays, we observed that knockdown of CCDC144NL-AS1 mediated inhibition of GC cell proliferation and migration were partially rescued by co-transfection with a miR-143$3 p$ inhibitor (Fig. 5a-k). These results suggested that the carcinogenic effect of CCDC144NL-AS1 was partially mediated by negative regulation of miR-143-3p.

\section{MAP3K7, a target gene of miR-143-3p, is regulated by CCDC144NL-AS1}

To explore the ceRNA network between CCDC144NLAS1, miR-143-3p, and its targets in GC, we used DIANA (http://diana.imis.athena-innovation.gr/DianaTools/ index.php), miRDB (http://www.mirdb.org/), miRTarBase (http://mirtarbase.mbc.nctu.edu.tw/php/index.php), TargetScan (http://www.targetscan.org/vert_71/) to predict putative targets of miR-143-3p, 21 genes may be the direct targets of miR-143-3p (Fig. 6a). Based on TCGA database, we selected four genes with relative higher expression in $\mathrm{GC}$ than in normal tissues as candidate target genes (Fig. S2A). Western blot indicated that MAP3K7 protein level 


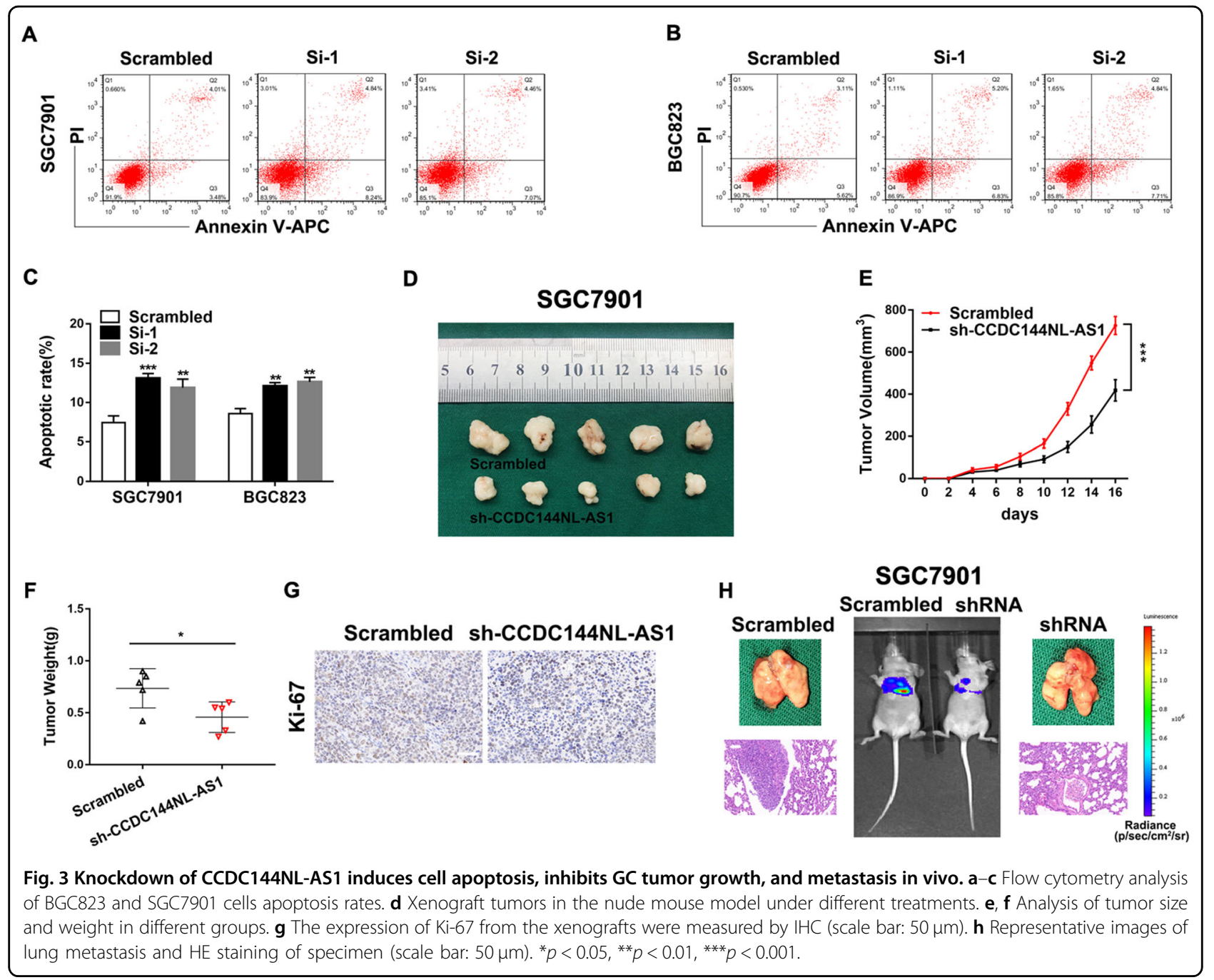

changed most obviously in SGC7901 cells transfected with miR-143-3p mimics or NC (Fig. S2B, C). A ceRNA network was constructed by Cytoscape (version 3.7.1) according to the data predicted by the above bioinformatics tools, in the network, we found that CCDC144NLAS1 was indeed correlated the predicted miR-143-3p and MAP3K7 (Fig. S3). So, MAP3K7 was identified as a potential target of miR-143-3p. A dual-luciferase reporter assay revealed that miR-143-3p markedly reduced the relative luciferase activity of the wild-type MAP3K7 $3^{\prime}$ UTR (Fig. 6b, c). TCGA database revealed that MAP3K7 was upregulated in GC samples (Fig. 6d). Consistent with TCGA analysis results, we verified higher MAP3K7 expression in $72 \mathrm{GC}$ tissues (Fig. 6e). A negative correlation was observed between MAP3K7 and miR-143-3p expression in 72 pairs of GC samples and a positive correlation was found between MAP3K7 and CCDC144NLAS1 expression (Fig. 6f, g). Subsequently, we transfected SGC7901 and BGC823 cells with miR-143-3p mimics, miR-143-3p inhibitor and their negative control group to investigate whether MAP3K7 is regulated by miR-143-3p, we observed that the MAP3K7 mRNA and protein level changed with upregulation or downregulation of miR143-3p, respectively (Fig. 6h, i, Fig. S4A). Further investigations indicated that downregulation of CCDC144NLAS1 positively affected MAP3K7 expression both at the mRNA and protein levels (Fig. 6j, k, Fig. S4B). For the rescue experiment, knockdown of miR-143-3p counteracted the corresponding decrease in MAP3K7 mRNA or protein expression induced by downregulation of CCDC144NL-AS1 in SGC7901 and BGC823 cells (Fig. 6l, $\mathrm{m}$, Fig. S4C). These results showed that MAP3K7 expression was primarily mediated by the regulation of miR-143-3p via CCDC144NL-AS1 at post-transcriptional level.

\section{Silencing MAP3K7 inhibits cell proliferation, induces GC cell apoptosis}

To explore the potential role of MAP3K7 in GC, we transfected SGC7901 and BGC823 cells with siRNA to 


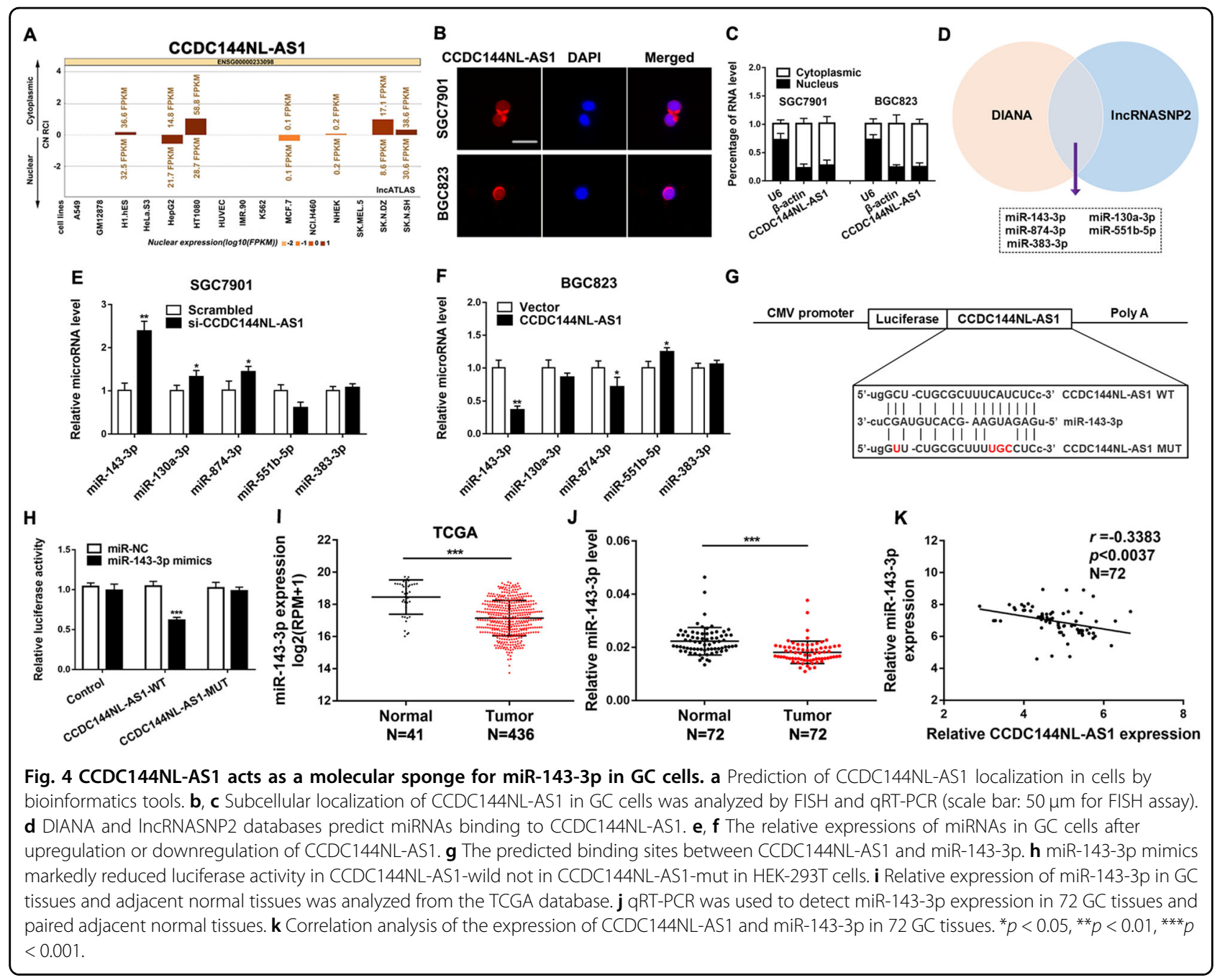

knockdown MAP3K7, which was identified at the mRNA and protein levels (Figs. S5A, B and S4D). CCK-8 assays and Edu assays indicated that knockdown of MAP3K7 could inhibit GC cell proliferation (Fig. S5C-G). To identify whether MAP3K7 knockdown could regulate tumor growth in vivo, SGC7901-shMAP3K7 and SGC7901-NC cells were subcutaneously inoculated into nude mice. We observed that the average size and weight of tumors generated by MAP3K7 knockdown group were markedly smaller (Fig. S5H-J). Flow cytometric assays also showed that knockdown of MAP3K7 induced GC cells apoptosis (Fig. S5K, L). These results suggested that silencing MAP3K7 inhibited cell proliferation, induced GC cell apoptosis.

The regulation of MAP3K7 on GC cells is mediated by miR143-3p

To further explore the biological interactions between MAP3K7 and miR-143-3p in GC, SGC7901, and BGC823 cells were co-transfected with si-MAP3K7, miR-143-3p inhibitor and their negative control groups. From CCK-8 assays, colony formation assays, transwell assays and wound healing assays, we observed that knockdown of MAP3K7 mediated inhibition of GC cell proliferation, migration and invasion were partially rescued by cotransfection with a miR-143-3p inhibitor (Fig. 7a-l). These results suggested that the carcinogenic effect of MAP3K7 was partially mediated by negative regulation of miR-143-3p.

\section{Discussion}

Large numbers of IncRNAs have been found to take part in the progression of tumor, including $\mathrm{GC}^{11,18,19}$. However, there are still many GC-related lncRNAs functional roles and molecular mechanisms that have not yet been clearly investigated. In the present study, we first reported lncRNA CCDC144NL-AS1, which was highly expressed in GC tissues and cell lines. The high expression of CCDC144NL-AS1 was related to larger tumor size and lymphatic metastasis. Besides, increased CCDC144NL- 


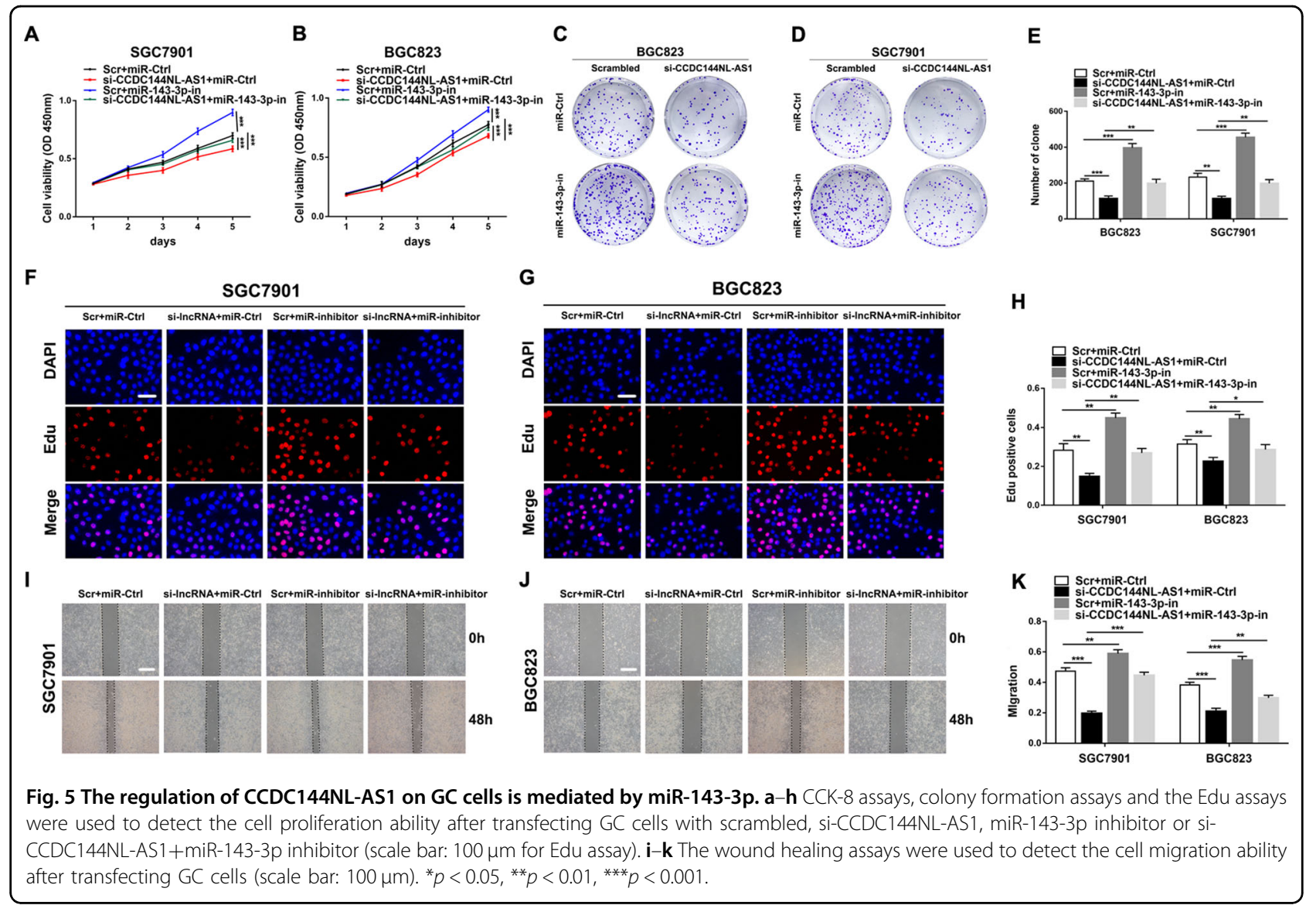

AS1 expression was associated with shorter OS time of GC patients. Further analysis indicated that silencing CCDC144NL-AS1 could inhibit cell proliferation, migration and invasion in $\mathrm{GC}$, and overexpression of CCDC144NL-AS1 yielded the opposite result. Meanwhile, in vivo experiments revealed that knockdown of CCDC144NL-AS1 alleviated lung metastasis and inhibited GC tumor growth. These results suggest that CCDC144NL-AS1 may exert a key function as an oncogene in GC.

The ceRNA hypothesis has attracted much attention in recent years ${ }^{15}$. Accumulating evidences suggests that lncRNA may act as a ceRNA for particular miRNAs to modulate the target genes of the miRNAs, including $\mathrm{GC}^{16,17,20}$. However, so far CCDC144NL-AS1 has never been reported to act as a ceRNA of miRNAs. In this study, we assessed the subcellular localization of CCDC144NLAS1 and observed that majority of CCDC144NL-AS1 were located in cytoplasm, which suggested that CCDC144NL-AS1 may function as a ceRNA for sponging miRNA. The subsequent bioinformatics analyses and luciferase reporter assays revealed that CCDC144NL-AS1 may bind with miR-143-3p. miR-143-3p has been reported to be downregulated in many tumors and functioned as a tumor suppressor, including $\mathrm{GC}^{21-23}$. We also found that miR-143-3p was markedly downregulated in GC tissues, and its expression level was negatively correlated with CCDC144NL-AS1. Besides, downregulation of miR143-3p could partially rescue the inhibition of GC cell proliferation mediated by knockdown of CCDC144NLAS1. So we think that CCDC144NL-AS1 may play a huge role in GC cells by sponging miR-143-3p during GC progression.

Generally, as a ceRNA, the function of lncRNAs depends on the miRNA target. Therefore, the target genes of miRNA are an important part of the ceRNA network $^{24,25}$. MAP3K7 was selected as a direct target of miR143-3p through bioinformatics analyses and luciferase reporter assays, which has never been reported. MAP3K7, which is known as TAK1(TGF- $\beta$-activated kinase-1), can be rapidly activated in response to TGF- $\beta$ signal transduction $^{26}$. MAP3K7 has been reported to participate in the development of various tumors ${ }^{27,28}$. With the deepening of research, we found that the expression of MAP3K7 was upregulated in GC samples. A negative or positive correlation was found among MAP3K7, miR-143$3 \mathrm{p}$ and CCDC144NL-AS1 expression in GC samples. Knockdown of MAP3K7 could inhibit cell proliferation, 


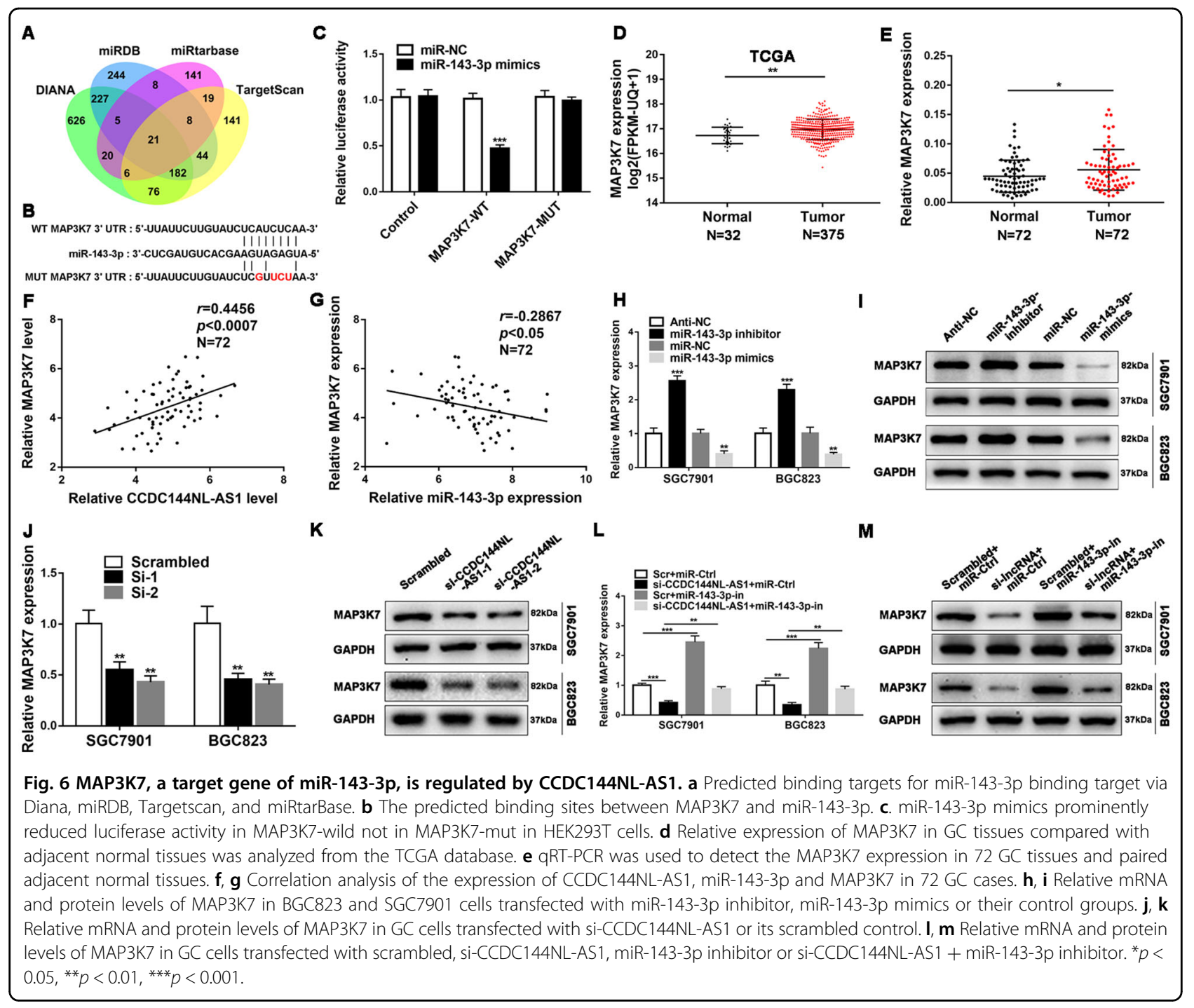

induces GC cell apoptosis. Besides, rescue experiments revealed that miR-143-3p could change proliferation, migration and invasion capabilities of tumor cells by interacting with MAP3K7 and exhibit regulative effects on GC. Thus, these results implied that CCDC144NL-AS1 functioned as a molecular sponge for miR-143-3p and upregulated the expression of its endogenous target MAP3K7 in GC. However, our study also has some limitations, the interaction between cytokines in cancer cells is very complex, we cannot rule out the role of CCDC144NL-AS1 in promoting GC through other pathways. We studied this signaling axis only in the GC cell lines SGC7901 and BGC823, it is quite necessary to extend relevant researches to other GC cell lines.

In summary, we identified a novel GC-associated lncRNA CCDC144NL-AS1. Through integrating clinical data and multipronged functional experiments, we illustrated that CCDC144NL-AS1 was upregulated in GC tissues and associated with poor prognosis. CCDC144NLAS1 upregulated MAP3K7 to promote GC cell proliferation, migration, and invasion by acting as a ceRNA that sponges miR-143-3p. Our results provide inspiration for further understanding of the mechanism of GC. In the future work, we will explore the mechanism of CCDC144NL-AS1 upregulation in GC, the correlation between CCDC144NL-AS1 and other miRNAs or proteins, which will further deepen our understanding of the pathogenesis of GC and make CCDC144NL-AS1 a potential novel diagnostic and therapeutic target for GC.

\section{Materials and methods Public data analysis}

A TCGA gene dataset named TCGA-STAD. htseq_fpkm-uq.tsv (including $372 \mathrm{GC}$ tissues and 35 normal tissues) with version number 07-20-2019 and A TCGA gene dataset named TCGA-STAD.mirna.tsv 


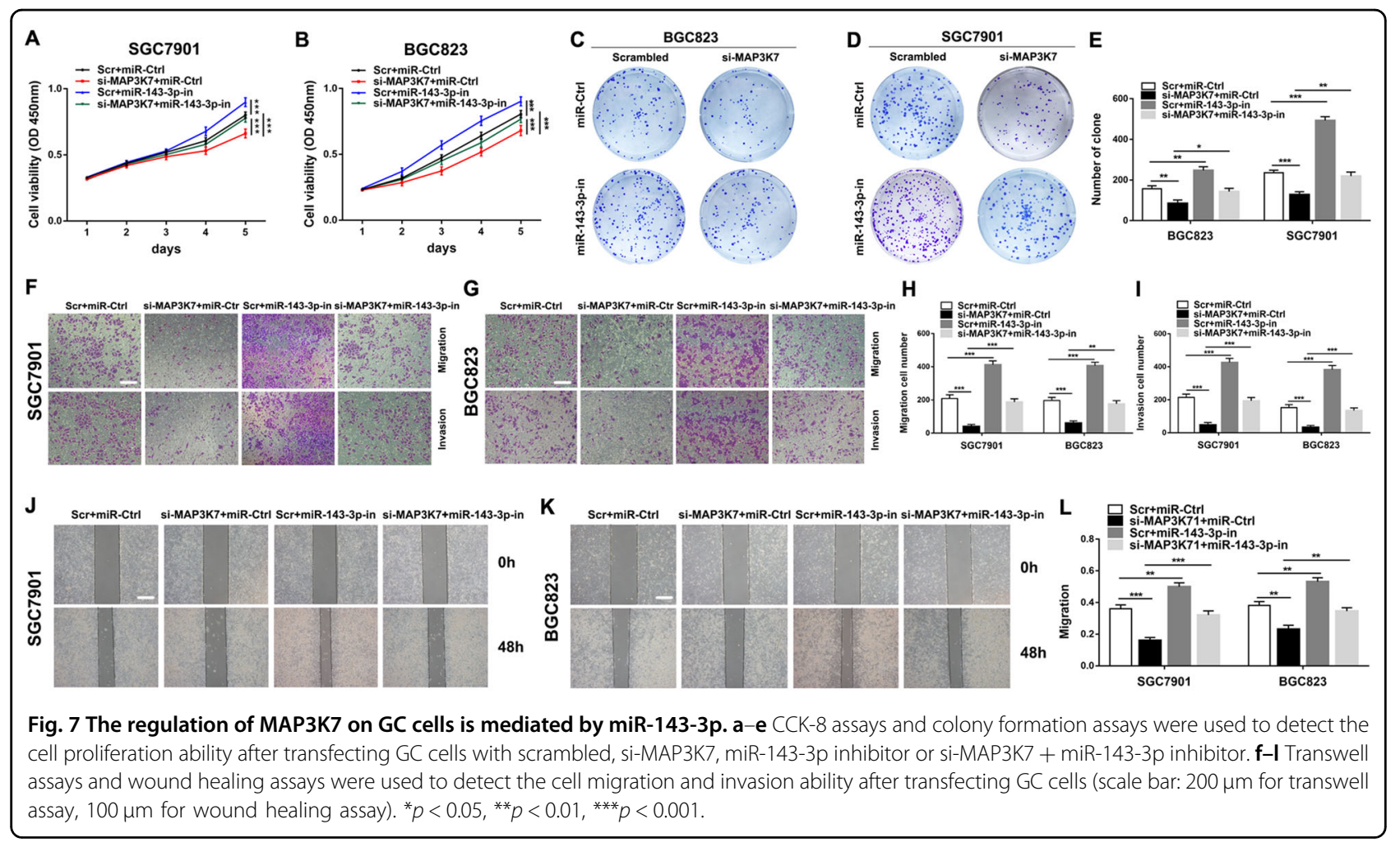

(including $436 \mathrm{GC}$ tissues and 41 normal tissues) with version number 07-20-2019 were downloaded from the UCSC cancer browser (https://xenabrowser.net/ datapages/). We obtained the normalized expression values of CCDC144NL-AS1, MAP3K7, and miR-143-3p from the above two files.

We have previously predicted miRNAs (miR-143-3p, miR-130a-3p, miR-874-3p, miR-551b-5p, and miR-383$3 p)$ that bind to CCDC144NL-AS1 and showed 21 mRNAs targeted by miR-143-3p. In order to build the ceRNA network, we used $\mathrm{R}$ software to predict the target mRNAs of remaining four miRNAs through the data from the following four databases: DIANA, miRDB, miRTarBase, and TargetScan. Finally, the lncRNA-miRNAmRNA regulatory network was constructed and visualized using Cytoscape software (version 3.7.1).

\section{Tissue specimens}

A total of $72 \mathrm{GC}$ tissues and matched normal tissues were obtained from patients with GC diagnosed by histopathological examination in the General Surgery Department of the First Affiliated Hospital of Nanjing Medical University (NMU). Immediately after excision, all samples were frozen with liquid nitrogen and stored at $-80^{\circ} \mathrm{C}$ until further use. This study was approved by the Ethics Committee of the First Affiliated Hospital of NMU. Written informed consent was signed before specimen collection.

\section{Cell culture}

Human GC cell lines (SGC7901, BGC823, AGS, MGC803) and the human normal gastric epithelial cell line GES-1 were purchased from the Institute of Biochemistry and Cell Biology of the Chinese Academy of Sciences (Shanghai, China). SGC7901, BGC823, MGC803 and AGS are all gastric adenocarcinoma cells. SGC7901 originated from lymph node metastasis in a 56-year-old woman with gastric adenocarcinoma. BGC823 was built from a 62-year-old patient with gastric cancer and is poorly differentiated. MGC803 was established from a 53year-old male patient with primary gastric poorly differentiated mucinous adenocarcinoma. AGS cell line originated from an untreated excised tumor fragment (http:// www.cellbank.org.cn/search.asp?a=1). SGC7901, BGC823, MGC803 and GES-1 cells were maintained in RPMI-1640 medium (Gibco, USA), AGS cells were cultured in F12K medium (Gibco, USA), supplemented with $10 \%$ fetal bovine serum (FBS, Gibco, USA) at $37{ }^{\circ} \mathrm{C}$ in a $5 \%$ $\mathrm{CO}_{2}$ incubator.

\section{Quantitative real-time reverse transcription polymerase chain reaction (qRT-PCR)}

LncRNA/mRNA was reverse-transcribed to cDNA with a Prime Script RT reagent Kit (Takara, China), a New Poly (A) Tailing Kit (Thermo Fisher Scientific, China) was used to reverse transcribe miRNAs into cDNA. cDNA was amplified with a 7500 Real-Time PCR System (Applied 
Biosystems, USA) with Universal SYBR Green Master Mix (Roche, Shanghai, China). $\beta$-actin or U6 was used as an endogenous control for mRNA and miRNA, respectively. All primer sequences are listed in Supplementary Table 2.

\section{Cell transfection}

Transfections were carried out using the Lipofectamine 3000 Reagent (Invitrogen, USA) following the manufacturer's protocol. The small interfering RNAs (siRNAs) targeting lncRNA/mRNA, miRNA mimics, miRNA inhibitors and their negative controls were designed and synthesized by GenePharma (Shanghai, China). Human CCDC144NL-AS1 transcript cDNA and short hairpin RNA (shRNA) directed against CCDC144NL-AS1 were cloned into the lentivirus vector CV084 and vector GV248, respectively (GenePharma, China). Stable cells were selected using puromycin. The sequences are listed in supplementary Table 2.

\section{Western blot}

Proteins were extracted and electrophoresed on a $10 \%$ SDS polyacrylamide gels, and then electrophoretically transferred onto a polyvinylidene fluoride (PVDF) membrane (Millipore, USA). The ECL chemiluminescent reagent (Beyotime Biotechnology, China) was used to detect chemical signals. The antibodies are listed in supplementary Table 2 .

\section{Cell counting kit-8 assay}

Thousand cells per well were seeded into 96-well plate and the cell proliferation rate was measured using Cell counting kit-8 assay (CCK-8) (Djingo, Japan) for 5 days. The absorbance at $450 \mathrm{~nm}$ was recorded using a standard microplate reader (Scientific MultiskanMK3, Thermo Scientific) according to the manufacturers' protocols.

\section{5-Ethynyl-2'-deoxyuridine (Edu) assay}

The Edu assay kit (RiboBio, China) was used to measure cell proliferation. Briefly, cells from different groups were treated with Edu and then stained by 4',6-diamid-ino-2phenylindole (DAPI). The images of Edu-positive cells were visualized under fluorescence microscope (Nikon, Japan).

\section{Colony formation}

Cells who were in six-well plates were incubated at $37^{\circ} \mathrm{C}$ in a $5 \% \mathrm{CO}_{2}$ incubator for two weeks. Then cells were stained, imaged and counted for the number of colonies.

\section{Transwell assay}

Transwell chambers with a membrane pore size of $8 \mathrm{~mm}$ were coated without or with Matrigel (BD
Biosciences, USA). A total of $2 \times 10^{4}$ cells were seeded into the upper chambers with serum-free medium, whereas medium containing 10\% FBS was used in the lower chamber. After incubation for $24 \mathrm{~h}$, the cells were fixed, stained, and counted using an inverted microscope.

\section{Wound healing assay}

Cells were seeded in 6-well plates and cultured to the subfusion state. Linear scratch wounds were created by $200 \mu \mathrm{l}$ sterile pipette tip and washed with phosphatebuffered saline (PBS). Images were captured at 0 and $48 \mathrm{~h}$, we calculated cell healing rates by the fraction of cell coverage across the line.

\section{Animal experiments}

Animal work was approved by the First Affiliated Hospital of Nanjing Medical University. For the tumor xenograft model, cells treated differently were subcutaneously inoculated into 4-week-old BALB/c nude mice (four or five mice for each group). Tumor volume was measured every 2 days. For about two weeks after injection, mice were euthanized and then the weight of xenografts was tested. For the metastasis model, cells treated differently were injected into the caudal vein of anesthetized nude mice (4 mice for each group). Mice were monitored using an IVIS imaging systems (Caliper Life Sciences, USA).

\section{Dual-Luciferase reporter assay}

The putative miR-143-3p binding sequences in CCDC144NL-AS1 or MAP3K7-3'-UTR and their mutant of the binding sites were synthesized and cloned to downstream of the luciferase gene in the pmirGLO luciferase vector (Promega, USA). HEK293T cells were cotransfected with the pmirGLO reporter plasmids and miR-143-3p mimics or miR-143-3p NC. The relative luciferase activity was measured with the Dual-Luciferase Reporter Assay System (Promega, USA).

\section{Flow cytometric analysis of apoptosis}

The apoptotic assay was conducted using an Annexin V-APC/PI Apoptosis Detection Kit (Multisciences, China) and analyzed with a flow cytometry (BD Biosciences, USA). The ratio of early and late apoptotic cells was detected to calculate the apoptotic rate.

\section{Isolation of nuclear and cytoplasmic RNA}

The isolation of nuclear and cytoplasmic RNA in BGC823 and SGC7901 cells were performed using the PARIS Kit (Invitrogen, USA) according to the manufacturer's instructions. Then the subcellular fractions were extracted and subjected to qRT-PCR. $\beta$-actin (cytoplasm control) and U6 (nucleus control)was used for normalization. 


\section{RNA fluorescent in situ hybridization}

A Cy3-labelled CCDC144NL-AS1 complementary DNA probe mix (RiboBio, China) was synthesized in vitro. fluorescent in situ hybridization (FISH) kit (RiboBio, China) was used to detect the subcellular localization of CCDC144NL-AS1 in BGC823 and SGC7901 cells according to the manufacturer's instructions. Images were observed with a confocal laser-scanning microscope (Olympus FV1000, Japan).

\section{Immunohistochemistry (IHC)}

All specimens were fixed and then embedded in paraffin. The paraffin-embedded sections were de-waxed in xylene and were rehydrated in graded alcohols. Then, specimens were incubated with primary antibodies Ki-67 (Abcam, USA) followed by secondary antibody conjugated with HRP. Subsequently, detection was conducted by $3,3^{\prime}$-diaminobenzidine and haematoxylin. The staining positivity was quantified in three different high-power fields of each section.

\section{Hematoxylin and eosin staining}

Hematoxylin and eosin (HE) was used to stain paraffinembedded lung sections of mice containing metastases. Sections were cut into 5- $\mu \mathrm{m}$ slices for pathological evaluation and observation under a microscope (Olympus, Japan).

\section{Statistical analysis}

Statistical analyses were performed using SPSS 22.0 (IBM, USA) and GraphPad Prism, version 6.00 (GraphPad Software, USA). Experimental data were shown as mean \pm standard deviation (SD),the significance of differences between groups was estimated by the two-tailed student $t$ test, Wilcoxon test, $\chi^{2}$ test or analysis of variance (ANOVA). Each experiment was repeated independently at least three times, $p<0.05$ was considered statistically significant.

\section{Acknowledgements}

This study was financially supported by the National Nature Science Foundation of China (81874219), the Natural Science Foundation of Jiangsu Province (BK20171505), the "Medical Zhong Dian Ren Cai Project" of Jiangsu Province (RC2011059), Li yang City's 2018 Annual research and development Plan Follows Nanjing Project (LC2019002).

\section{Conflict of interest}

The authors declare that they have no conflict of interest.

\section{Publisher's note}

Springer Nature remains neutral with regard to jurisdictional claims in published maps and institutional affiliations.
Supplementary Information accompanies this paper at (https://doi.org/ 10.1038/s41419-020-02740-2).

Received: 5 March 2020 Revised: 23 June 2020 Accepted: 29 June 2020

Published online: 09 July 2020

\section{References}

1. Chen, W. et al. Cancer statistics in China, 2015. CA Cancer J. Clin. 66, 115-132 (2016).

2. Bray, F. et al. Global cancer statistics 2018: GLOBOCAN estimates of incidence and mortality worldwide for 36 cancers in 185 countries. CA Cancer J. Clin. 68, 394-424 (2018)

3. Van Cutsem, E. \& Ducreux, M. Colorectal and gastric cancer in 2015: The development of new agents and molecular classifications. Nat. Rev. Clin. Oncol. 13, 69-70 (2016)

4. Yu, J. et al. Effect of laparoscopic vs open distal gastrectomy on 3-year diseasefree survival in patients with locally advanced gastric cancer: the CLASS-01 Randomized Clinical Trial. JAMA 321, 1983-1992 (2019).

5. Shen, L. et al. Management of gastric cancer in Asia: resource-stratified guidelines. Lancet Oncol. 14, e535-e547 (2013).

6. Consortium, E. P. An integrated encyclopedia of DNA elements in the human genome. Nature 489, 57-74 (2012).

7. Nagano, T. \& Fraser, P. No-nonsense functions for long noncoding RNAs. Cell 145, 178-181 (2011).

8. Mercer, T. R. \& Mattick, J. S. Structure and function of long noncoding RNAs in epigenetic regulation. Nat. Struct. Mol. Biol. 20, 300-307 (2013).

9. $\mathrm{Xu}, \mathrm{T}$. P. et al. SP1-induced upregulation of the long noncoding RNA TINCR regulates cell proliferation and apoptosis by affecting KLF2 mRNA stability in gastric cancer. Oncogene 34, 5648-5661 (2015).

10. Mondal, T. et al. Sense-Antisense IncRNA Pair Encoded by Locus 6 p22.3 Determines Neuroblastoma Susceptibility via the USP36-CHD7-SOX9 Regulatory Axis. Cancer Cell. 33, 417-434 e417 (2018).

11. De Troyer, L. et al. Stress-induced IncRNA LASTR fosters cancer cell fitness by regulating the activity of the U4/U6 recycling factor SART3. Nucleic Acids Res. 48, 2502-2517 (2020)

12. Tang, J. et al. LnCRNA GLCC1 promotes colorectal carcinogenesis and glucose metabolism by stabilizing c-Myc. Nat. Commun. 10, 3499 (2019).

13. Wang, $Y$. et al. LncRNA HAND2-AS1 promotes liver cancer stem cell selfrenewal via BMP signaling. EMBO J. 38, e101110 (2019).

14. Zhang, E. et al. A novel long noncoding RNA HOXC-AS3 mediates tumorigenesis of gastric cancer by binding to YBX1. Genome Biol. 19, 154 (2018)

15. Salmena, L., Poliseno, L., Tay, Y., Kats, L. \& Pandolfi, P. P. A ceRNA hypothesis: the Rosetta Stone of a hidden RNA language? Cell 146, 353-358 (2011)

16. Chen, $X$. et al. Long noncoding RNA LINC01234 functions as a competing endogenous RNA to regulate CBFB expression by sponging miR-204-5p in gastric cancer. Clin. Cancer Res. 24, 2002-2014 (2018).

17. Zheng, Z. Q. et al. Long noncoding RNA FAM225A promotes nasopharyngeal carcinoma tumorigenesis and metastasis by acting as ceRNA to sponge miR-590-3p/miR-1275 and upregulate ITGB3. Cancer Res. 79, 4612-4626 (2019).

18. Xiao, G. et al. The long noncoding RNA TTTY15, which is located on the $Y$ chromosome, promotes prostate cancer progression by sponging let-7. Eur. Urol. 76, 315-326 (2019).

19. Xu, T. P. et al. KLF5 and MYC modulated LINC00346 contributes to gastric cancer progression through acting as a competing endogeous RNA and indicates poor outcome. Cell Death Differ. 26, 2179-2193 (2019).

20. Credendino, S. C. et al. A ceRNA circuitry involving the long noncoding RNA Klhl14-AS, Pax8, and Bcl2 drives thyroid carcinogenesis. Cancer Res. 79, 5746-5757 (2019)

21. Jin, Y. P. et al. miR-143-3p targeting of TTGA6 suppresses tumour growth and angiogenesis by downregulating PLGF expression via the PI3K/AKT pathway in gallbladder carcinoma. Cell Death Dis. 9, 182 (2018). 
22. He, Z. et al. MiR-143-3p functions as a tumor suppressor by regulating cell proliferation, invasion and epithelial-mesenchymal transition by targeting QKI5 in esophageal squamous cell carcinoma. Mol. Cancer 15, 51 (2016).

23. Wu, X. L. et al. MicroRNA-143 suppresses gastric cancer cell growth and induces apoptosis by targeting COX-2. World J. Gastroenterol. 19, 7758-7765 (2013).

24. Karreth, F. A. \& Pandolfi, P. P. ceRNA cross-talk in cancer: when ce-bling rivalries go awry. Cancer Discov. 3, 1113-1121 (2013).

25. Tay, Y., Rinn, J. \& Pandolfi, P. P. The multilayered complexity of ceRNA crosstalk and competition. Nature 505, 344-352 (2014).
26. Yamaguchi, K. et al. Identification of a member of the MAPKKK family as a potential mediator of TGF-beta signal transduction. Science 270, 2008-2011 (1995).

27. Zehavi, L. et al. MiR-377 targets E2F3 and alters the NF-kB signaling pathway through MAP3K7 in malignant melanoma. Mol. Cancer 14, 68 (2015).

28. Washino, S. et al. Loss of MAP3K7 sensitizes prostate cancer cells to CDK1/2 inhibition and DNA Damage by disrupting homologous recombination. Mol. Cancer Res. 17, 1985-1998 (2019). 